\title{
Soporte social percibido en cuidadores familiares de personas con enfermedad crónica
}

\author{
Social support perceived in family caregivers of people with chronic disease
}

Sandra Lucrecia Romero-Guevara ${ }^{*}$ orcid.org/0000-0002-6845-3159

Bertha Lucia Correa ${ }^{1}$ orcid.org/0000-0002-4336-3368

Fabio Alberto Camargo-Figuera ${ }^{1}$ orcid.org/0000-0001-6070-9327

1 Escuela de Enfermería, Universidad Industrial de Santander, Bucaramanga, Colombia

\section{Resumen}

Introducción: El aumento de las enfermedades crónicas ha puesto en evidencia la problemática entre la oferta y demanda de los servicios de salud, generando el desplazamiento de cuidados al hogar y sobrecarga a los cuidadores familiares. Objetivo: determinar el soporte social percibido por los cuidadores familiares de personas con enfermedad crónica. Materiales y Métodos: Se realizó un estudio de corte trasversal, se aplicó el instrumento: Inventario de Soporte Social en Enfermedad Crónica de Hilbert a 129 cuidadores de personas con enfermedad crónica atendidos en un hospital de tercer nivel de atención. Resultados: Los cuidadores continúan siendo principalmente mujeres (89,9\%). El puntaje promedio de la escala fue de 110,7, desviación estándar $=38,1$, observándose diferencias significativas en la escolaridad $(p=0,030)$, el estrato socioeconómico $(p=0,013)$ y el alojamiento conjunto al paciente $(\mathrm{p}=0,010)$. Conclusión: Los cuidadores reportaron estar poco satisfechos con el soporte social percibido y esta percepción estuvo relacionada con las condiciones socioeconómicas de las personas.
\end{abstract}

Palabras clave: Apoyo social; cuidadores; enfermedad cronica. (Fuente: DeCS, Bireme).

\begin{abstract}
Introduction: The increase in chronic diseases has put in evidence the problem between the supply and demand for health services, generating the displacement of care to home and the overload to family caregivers. Objective: To determine the social support perceived by family caregivers of people with chronic disease. Materials and Methods: A transversal study was conducted and the Inventory of Social Support on Chronic Disease of Hilbert instrument was used with 129 family caregivers of people with chronic disease seen in a hospital of third level of attention. Results: The caregivers are primarily women (89.9\%). The average score of the scale was of $\$ 110.7$, standard deviation $=38.1$. Significant differences were found in the schooling $(p=0.030)$, socioeconomic status $(p=0.013)$ and the housing to the patient $(p=0.010)$. Conclusion: The caregivers reported to be unhappy with perceived social support and this perception was related to the socio-economic conditions of the people.
\end{abstract}


Keywords: Social support; caregivers; chronic disease. (Source: DeCS, Bireme).

\section{Introducción}

El direccionamiento de los servicios de salud hacia la implementación de estrategias en salud pública ha representado grandes avances en el control de la natalidad y de muchas enfermedades infecciosas, por lo tanto, ha favorecido el aumento en la expectativa de vida de la población y de manera particular en la población adulta mayor (mayor de 60 años), aspecto que aumenta la vulnerabilidad de presentar factores de riesgo, perder la autonomía o la funcionalidad a causa de las enfermedades crónicas ${ }^{1}$.

De otra parte, los avances tecnológicos y el estilo de vida actual han favorecido hábitos poco saludables (tabaquismo, sedentarismo, consumo de dietas ricas en contenido graso, sodio, carbohidratos, etc.), aspectos que contribuyen al incremento en la prevalencia de las enfermedades no trasmisibles, tales como las enfermedades cardiovasculares, el cáncer y las enfermedades respiratorias crónicas, que han ido en aumento según las estadísticas mundiales de los últimos años ${ }^{2-4}$.

En relación con lo anterior, los sistemas de salud enfrentan un gran desequilibrio entre una oferta insuficiente y la alta demanda de los servicios de salud, lo cual ha conllevado a la implementación de estrategias, entre las cuales se encuentran el traslado de los cuidados al hogar y la participación activa de familiares y cuidadores informales en la realización de actividades para la satisfacción de necesidades básicas (alimento, higiene, baño, vestido, entre otras.), así como intervenciones para dar cumplimiento al régimen terapéutico (medicamentos, dieta prescrita, actividad física, control de factores de riesgo, etc.), e incluso la práctica de cuidados especializados como el cuidado de heridas, traqueostomía, aspiración de secreciones, gastrostomía y sondajes, entre otros ${ }^{5-7}$.
Al respecto, es importante considerar que los cuidados que una persona con enfermedad crónica requiere para su bienestar, demandan tiempo, dedicación y esfuerzo físico para el cuidador, lo cual representa una carga física y emocional para los cuidadores familiares, que se hace más evidente cuando los enfermos crónicos son altamente dependientes, demandantes de cuidado y cuando los cuidadores no reciben la preparación necesaria y no cuentan con el apoyo para ejercer los cuidados de manera adecuada y segura ${ }^{8,9}$.

En cuanto al soporte social, es un constructo multifactorial que ha sido estudiado y definido por diferentes autores. En general ha sido considerado como un elemento esencial en la interacción en los seres humanos y un factor protector en situaciones de crisis ${ }^{10}$. Según Hilbert el soporte social es de vital importancia para los cuidadores de personas con enfermedad crónica. El contar con una buena interacción personal, guía, retroalimentación, ayuda tangible, interacción social, permite a los cuidadores tener una mejor experiencia al cuidar ${ }^{11}$.

Está claro que los cuidadores tienen un papel protagónico en el cuidado de las personas con enfermedad crónica y que requieren de redes de apoyo que les brinden soporte social, dentro de las cuales se encuentra el sistema y el equipo de salud. Dentro de los miembros del equipo de salud, los profesionales de enfermería son líderes en brindar cuidado a usuarios y poblaciones vulnerables y en el proceso de cuidar, son responsables de identificar las necesidades de los mismos y de promover la potenciación de roles por medio de la preparación para las intervenciones de cuidado, que son aspectos claves y útiles para entender y apoyar las necesidades del cuidador dentro del entorno familiar y comunitario12,13. De acuerdo a lo planteado, el presente trabajo tiene el objetivo de determinar el soporte social percibido por los cuidadores familiares de personas con enfermedad crónica. 


\section{Materiales y métodos}

Estudio de corte transversal descriptivo que caracteriza a los cuidadores familiares y el soporte social percibido por ellos, adicionalmente el estudio cuenta con un componente analítico y exploratorio que permite evaluar algunas características sociodemográficas de los cuidadores y la relación con el soporte social percibido.

La población de estudio fueron los cuidadores familiares de pacientes con enfermedades crónicas atendidos en una institución de salud de tercer nivel de complejidad en los servicios de medicina interna, hemato-oncología y pediatría, y en la consulta externa de medicina interna. La muestra estuvo conformada por 129 cuidadores familiares mayores de 18 años, alfabetos y que no recibían remuneración económica por la labor de cuidar, quienes fueron seleccionados por conveniencia hasta completar la muestra. Los cuidadores fueron abordados en los servicios en mención durante un periodo de tres meses, durante el proceso se verificaban criterios de inclusión, se les invitaba a participar y si aceptaban se les aplicaba consentimiento informado por escrito. El tamaño de muestra consideró un nivel de confianza del 95\%, un poder del $80 \%$, una desviación estándar de 35 para el puntaje de soporte social, para detectar diferencias de mínimo 15 puntos en la escala de soporte social.

Para medir el soporte social percibido se empleó el instrumento Inventario de Soporte Social en Enfermedad Crónica (ISSEC) de Hilbert ${ }^{11}$, el cual está integrado por 38 variables, que a su vez determinan 5 aspectos o subescalas: 1) Interacción personal, constituye el apoyo de tipo emocional requerido para el adecuado afrontamiento en diversas situaciones de enfermedad crónica (10 ítems); 2) Guía, se relaciona con la ayuda que percibe el cuidador en cuanto a educación e información (14 ítems); 3) Retroalimentación, tiene que ver con el apoyo en las acciones (5 ítems); 4) Ayuda tangible, es el apoyo material, la ayuda en las tareas físicas y en las labores desempeñadas por el cuidador (4 ítems) y finalmente 5) Interacción social, que hace referencia al apoyo que reciben los cuidadores para rescatar sus relaciones con otros grupos, familias e instituciones que respondan a sus necesidades de relajación y esparcimiento (5 ítems). El instrumento original presenta una concordancia del $100 \%$, con una fiabilidad medida con un alfa de Cronbach de 0,80 y para las subescalas entre 0,84 y 0,9411 .

Para el presente estudio se excluyó la categoría de ayuda tangible con sus 4 ítems (dinero, transporte, regalos y reemplazo en tareas), dado que no corresponde al personal de enfermería, ni fue propósito del estudio brindar este tipo de apoyo. Finalmente se aplicaron 34 ítems del instrumento, con un rango posible de puntaje de 34 a 204, en donde a mayor puntaje mayor soporte social percibido. Cada ítem se mide por medio de una escala tipo Likert con 6 opciones de respuesta: insatisfecho, algo insatisfecho, parcialmente satisfecho, algo satisfecho, satisfecho y muy satisfecho.

Adicionalmente, fueron evaluadas algunas variables de interés que permitieran caracterizar la muestra y evaluar diferencias del soporte social. Entre estas variables están características socio-demográficas como género, edad del cuidador, edad de la persona enferma, estado civil, ocupación y estrato socioeconómico, además características relacionadas con el cuidado como fueron el tiempo como cuidador, cuidado desde el diagnóstico, alojamiento conjunto, contar con apoyo económico, emocional, asistencial y espiritual, relación con la persona enferma y grupo de diagnóstico médico.

Los datos fueron digitados en dos bases independientes y validadas en el programa EpiInfo $6.04 \mathrm{~d}^{14} \mathrm{y}$ fueron analizados en Stata $12^{15}$. Se calcularon frecuencias absolutas y relativas, para la descripción de resultados de variables categóricas y medidas de tendencia central y dispersión (según su distribución) para variables continuas. En el análisis bivariado fueron comparadas las medias y desviaciones estándar del puntaje de la escala de soporte social entre cada una de las categorías de las variables, para ello fueron utilizadas las pruebas $t$ de Student, ANOVA y prueba de tendencia lineal. 
Se consideró diferencias estadísticamente significativas aquellas cuyo valor de $\mathrm{p}$ fuera menor de 0,05. La normalidad del puntaje total de soporte social fue evaluada por medio de pruebas gráficas y estadísticas, una de ellas la prueba de Shapiro Wilk. Complementariamente fue calculado el coeficiente Alpha de Cronbach como medida de la confiabilidad o consistencia interna del instrumento, se estimaron coeficientes para la escala y para cada una de sus dimensiones.

\section{Consideraciones éticas}

La investigación mantuvo los parámetros éticos establecidos en la Resolución 008430 de 1993, del Ministerio de Salud de Colombia ${ }^{16}$. El estudio se consideró de riesgo mínimo para los participantes y se solicitó el consentimiento informado a cada cuidador. Así mismo, la información obtenida fue manejada bajo los principios éticos para investigación en salud ${ }^{17}$, contando con la aprobación por parte del Comité de Ética de la Facultad de Salud de la Universidad Industrial de Santander.

\section{Resultados}

El instrumento de soporte social presentó muy buena consistencia interna. El coeficiente Alpha de Cronbach para la escala general fue de 0,97, para las dimensiones de interacción personal 0,91 , guía 0,95 , retroalimentación 0,91 y para interacción social 0,90.

El análisis de los datos permitió demostrar que los 129 cuidadores obtuvieron una media en soporte social de 110,7 , con una desviación estándar (DE) de 38,1, un puntaje mínimo de 34 y máximo de 198. El puntaje total del soporte social presentó una distribución normal de acuerdo a la prueba de Shapiro Wilk ( $p>0,05)$. En cuanto a las dimensiones los cuidadores presentaron los siguientes resultados: 1)
Interacción personal: puntaje promedio de 32.7, $\mathrm{DE}=11,5$, rango $10-60 ; 2$ ) Guía: puntaje promedio de $47,5, \mathrm{DE}=17,1$, rango $14-84 ; 3$ ) Retroalimentación: puntaje promedio de 16.2, $\mathrm{DE}=6,7$, rango 5-30; 4) Interacción social: puntaje promedio de $14,3, \mathrm{DE}=6,5$, rango $5-30$.

En cuanto a las características demográficas, se pudo evidenciar que el papel de cuidador sigue siendo un rol predominantemente femenino con un $89.9 \%$ del total de la muestra; de otra parte, los cuidadores de este estudio fueron principalmente hijos(as) de la persona enferma en un $41,1 \%$ y padres en un $26,4 \%$, cuyas edades estuvieron en el rango de 36 a 59 años $(59,4 \%)$ y menores de 35 años $(31,2 \%)$. Por su parte el rango de edad de la persona enferma fue mayor que la del cuidador en un $67,4 \%$.

Al caracterizar las condiciones socioeconómicas de los cuidadores, se encontró que se distribuyen de manera equitativa en los estratos socioeconómicos 1 (34,9\%), 2 (32,5\%) y 3 (32,5\%), los niveles de escolaridad más prevalentes fueron primaria incompleta $(27,9 \%)$, secundaría incompleta $(20,9 \%)$ y completa $(20,9 \%)$. En cuanto al estado civil y la ocupación, el 54.3\% viven en unión estable y el $55 \%$ se dedican principalmente a las labores del hogar.

Al evaluar la relación de las características sociodemográficas con el soporte social percibido por los cuidadores, sólo se encontró diferencias estadísticamente significativas en la escolaridad $(p=0,03)$ y en el estrato socioeconómico $(\mathrm{p}=0,013)$, evidenciándose que los cuidadores con secundaria incompleta perciben mayor soporte social en comparación con los otros rangos de escolaridad. En cuanto al estrato socio-económico hubo una tendencia lineal significativa, ya que al aumentar el estrato socioeconómico disminuye el puntaje de soporte social (Tabla 1). 
Tabla 1. Descripción sociodemográfica y soporte social. Bucaramanga, Colombia, 2013 ( $\mathrm{n}=129)$

\begin{tabular}{|c|c|c|c|c|}
\hline Variable & n & $\%$ & \multicolumn{2}{|c|}{$\begin{array}{l}\text { Soporte social } \\
\text { Media } \quad \text { DE }\end{array}$} \\
\hline Soporte social & & & & \\
\hline Total de la muestra & 129 & 100 & 110,7 & 38,1 \\
\hline Género & & & & \\
\hline Masculino & 13 & 10,1 & 120,1 & 32,2 \\
\hline Femenino & 116 & 89,9 & 109,6 & $\begin{array}{r}38,7 \\
p=0,350^{*}\end{array}$ \\
\hline Edad del cuidador(a) & & & & \\
\hline Menor de 35 años & 40 & 31,2 & 118,7 & 38,5 \\
\hline Entre 36 y 59 años & 76 & 59,4 & 106,3 & 36,3 \\
\hline Mayor de 60 años & 12 & 9,4 & 112,3 & $\begin{array}{r}47,9 \\
\mathrm{p}=0,251^{*}\end{array}$ \\
\hline Edad de la persona enferma & & & & \\
\hline Mayor que rango de edad del cuidador(a) & 87 & 67,4 & 107,6 & 38,6 \\
\hline Menor o mismo rango de edad del cuidador(a) & 42 & 32,6 & 116,9 & $\begin{array}{r}36,8 \\
\mathrm{p}=0,196^{*}\end{array}$ \\
\hline Relación con la persona enferma & & & & \\
\hline Esposo(a) & 15 & 11,6 & 118,3 & 32,9 \\
\hline Madre/padre & 34 & 26,4 & 120,6 & 38,5 \\
\hline Hijo(a) & 53 & 41,1 & 108,2 & 39,9 \\
\hline Otro & 27 & 20,9 & 98,7 & $\begin{array}{r}34,4 \\
\mathrm{p}=0,120^{* *}\end{array}$ \\
\hline Escolaridad & & & & \\
\hline Primaria incompleta & 18 & 14,0 & 118,1 & 34,4 \\
\hline Primaria completa & 36 & 27,9 & 100,8 & 42,2 \\
\hline Secundaria incompleta & 27 & 20,9 & 127,2 & 40,2 \\
\hline Secundaria completa & 27 & 20,9 & 99,8 & 29,1 \\
\hline Técnica o superior & 21 & 16,3 & 113,9 & $\begin{array}{r}34,9 \\
\mathrm{p}=0,030^{* *}\end{array}$ \\
\hline Estado civil & & & & \\
\hline Con unión estable & 70 & 54,3 & 112,4 & 37,2 \\
\hline Sin unión estable & 59 & 45,7 & 108,6 & $\begin{array}{r}39,4 \\
\mathrm{p}=0,579^{*}\end{array}$ \\
\hline Ocupación & & & & \\
\hline Hogar & 71 & 55,0 & 114,4 & 41,5 \\
\hline Empleado(a) & 25 & 19,4 & 104,8 & 33,6 \\
\hline Trabajador(a) independiente & 18 & 14,0 & 104,8 & 36,8 \\
\hline Otro & 15 & 11,6 & 109,7 & $\begin{array}{r}30,5 \\
\mathrm{p}=0,637^{* *}\end{array}$ \\
\hline Estrato socioeconómico & & & & \\
\hline 1 & 45 & 34,9 & 121,5 & 41,7 \\
\hline 2 & 42 & 32,5 & 108,2 & 38,9 \\
\hline 3 o superior & 42 & 32,5 & 101,5 & $\begin{array}{r}30,6 \\
p=0,013 \dagger\end{array}$ \\
\hline
\end{tabular}

DE: Desviación estándar * Prueba t de Student ** ANOVA † Prueba de tendencia lineal

\section{Discusión}

Múltiples estudios describen la importancia del soporte social para un adecuado afrontamiento y desarrollo del rol del cuidador ${ }^{18}$, teniendo en cuenta que la condición de ser cuidador de una persona con enfermedad crónica, representa un reto para las capacidades físicas, emocionales, sociales, espirituales y económicas, para la 
persona o familia que asume el rol de cuidar y responder por la salud y el bienestar de otro ser humano. De ahí que dicha labor represente una carga que si no es controlada puede traer consecuencias poco favorables tanto para la persona enferma como para el cuidador, debido a los sentimientos de frustración e impotencia que puedan conducir a baja autoestima, depresión y a estrés por sobrecarga.

Los principales hallazgos de este estudio evidencian un soporte social promedio de 110,7 (DE 38,1), con un rango posible entre 34 y 204 , lo cual refleja que los cuidadores familiares de personas con enfermedad crónica se encuentran entre poco y algo satisfechos con el soporte social que perciben. Al detallar por cada una de las dimensiones los cuidadores perciben entre poco y algo de satisfacción en las dimensiones Interacción Personal, con un puntaje promedio de 32,7 (rango de 10 a 60); guía, con un puntaje promedio de 47,5 (rango de 14 a 84) y retroalimentación con un puntaje promedio de 16,2 (rango de 5 a 30). La dimensión de Interacción social obtuvo el promedio más bajo 14,3 (rango de 5 a 30), lo cual indica que los cuidadores se encuentran entre algo insatisfechos y poco satisfechos con la retroalimentación recibida por las enfermeras en el proceso de cuidar a su familiar enfermo.

Otros hallazgos encontrados en este estudio fueron, que a mayor nivel socioeconómico menor soporte social percibido, además que las personas con nivel de escolaridad secundaria incompleta perciben mayor soporte social y que los cuidadores que conviven con la persona que cuidan perciben mayor soporte social que los que viven en domicilios diferentes.

En el marco de este estudio, al igual que lo reportado en la literatura ${ }^{19}$, el género de los cuidadores familiares son en su gran mayoría mujeres, amas de casa, hijas de la persona cuidada y que se encuentran en un rango de edad media, estas variables son importantes de mencionar debido a que varios artículos han descrito que la población femenina es más propensa a sufrir de estrés dadas sus condiciones de madre, esposas, administradoras del hogar y sumado a lo anterior la ardua y difícil experiencia de cuidar a un ser querido que demandará su atención y su apoyo ${ }^{6}$.

Igualmente se puede evidenciar que la familia es la principal fuente de apoyo social con que contamos los seres humanos y en especial en situaciones de dificultad, como lo es afrontar un proceso de enfermedad y en especial si es una situación de cronicidad. El soporte familiar permite al enfermo crónico afrontar con éxito los problemas a que da lugar la enfermedad, donde la cuidadora principal aporta apoyo instrumental, afectivo y emocional ${ }^{20,21}$.

Los resultados permitieron observar que los cuidadores de este estudio con menor nivel socioeconómico y escolaridad incompleta, presentaron un puntaje mayor en la escala de Inventario de Soporte Social en Enfermedad Crónica, lo cual se aleja de lo encontrado en la literatura, debido a que las características de baja escolaridad y residir en estratos bajos son condiciones que promueven empleos de baja remuneración o el difícil acceso inclusive de un sistema de salud de calidad, escenario desfavorable debido a que las enfermedades crónicas representan un alto costo para la familia ${ }^{3,6}$.

En relación a lo anterior, múltiples autores han descrito las altas implicaciones financieras que trae una enfermedad crónica como consecuencia del tiempo que dedican los cuidadores a las labores del cuidado informal y a la cantidad de insumos, medicamentos, equipos, procedimientos, entre otros; que son necesarios para su manejo y que en muchos casos no son apoyados o subsidiados y que conllevan a situaciones de frustración e impotencia por parte de los personas enfermas y sus familias ${ }^{12,22-24}$.

Por otra parte, la Organización Mundial de la Salud en su reporte general de prevención de enfermedades crónicas en 2010 afirma que el incremento de estas enfermedades representa una amenaza creciente y que constituye una causa subestimada de pobreza y dificultad para el desarrollo económico de muchos países, teniendo en cuenta que el rol de cuidador 
requiere que la persona que lo ejerza invierta de su tiempo alrededor de 12 horas al día o más, lo cual representa para la familia la pérdida de ingresos económicos tanto por la persona enferma como por el cuidador 25 .

Otro aspecto importante de contemplar es el hecho de que convivir con la persona enferma puede conllevar a mayor percepción de soporte social, un aspecto que puede explicar lo anterior, es que los seres humanos cuando experimentan situaciones de adversidad y en particular en las comunidades con bajos recursos, sin opciones de estabilidad laboral, poseen una gran empatía o solidaridad entre sus integrantes. Es así que los vecinos, amigos, familiares e integrantes del equipo de salud, aunque no puedan favorecer el "Apoyo tangible", si pueden representar un apoyo en lo emocional, espiritual y en lo educativo; tal y como fue evidenciado por Quero, quien refiere que "el apoyo personal para el cuidador radica esencialmente en la necesidad de unas palabras de consuelo o una mirada de animó" 26 .

Al analizar específicamente los resultados del soporte social con los reportados en otros estudios, el promedio del soporte social se encuentra inferior al reportado en otras regiones del país como en los estudios de Herrera et al.27, realizado en Cartagena, donde el promedio alcanzó 148,97 (DE 33,94) y en el de Barrera et al.28, realizado en Bogotá, en el cual el promedio fue de 136,18 (DE 40,41), valores que permiten concluir que los cuidadores en estos estudios perciben mayor satisfacción con el soporte social que reciben al cuidar. Es importante aclarar que en los mismos, no se incluyó la dimensión de ayuda tangible, al igual que el presente trabajo.

En cuanto a las categorías o dimensiones del soporte social, los cuidadores de los estudios en mención muestran buen nivel de satisfacción en el soporte que reciben tanto en interacción personal, guía, retroalimentación e interacción social, comparado con los resultados del presente estudio donde se reporta un grado parcial de satisfacción. Se hace evidente la diferencia hallada en la categoría de interacción social dado que los estudios de Herrera et al., muestran puntajes de 21,95 y 19,4227,28, en comparación con 14,3 en este estudio, valor que refleja cierto grado de insatisfacción con el soporte recibido en esta categoría.

Estos resultados pueden ser explicados por múltiples aspectos, considerando que el soporte social es un constructo multifactorial y entendiendo que las enfermedades crónicas son procesos dinámicos vividos por seres humanos en constante trasformación. Al respecto Vargas refiere "Es relevante considerar que la salud del individuo, el cuidador y la familia en situación de enfermedad crónica es el resultado de múltiples transiciones en el tiempo, que reflejan los patrones de vida y experiencia con relación a la enfermedad" es así que cada persona y su cuidador familiar "se encuentra inmerso en una situación de enfermedad crónica única que refleja lo que se ve, siente y se percibe en su vida"12. De otra parte, los cuidadores del presente estudio a diferencia del estudio de Herrera et al. y Barrera et al., en el momento de la medición estaban viviendo el proceso de la hospitalización o quimioterapia de su familiar, lo cual constituye un factor de tensión al sacarlos de la rutina del cuidado y al aumentar los costos económicos asociados a los traslados y a la estancia hospitalaria, efectos que han sido documentados en la literatura ${ }^{24}$.

Consideramos fortalezas de este estudio el poder determinar el soporte social percibido por los cuidadores de personas con enfermedad crónica durante todo el proceso de cuidar, y en especial porque en el momento de la medición se encontraban en un evento de hospitalización o quimioterapia, que son situaciones a las que muchos cuidadores deben enfrentarse en su rol de cuidadores. Además, se logró explorar algunos factores que influyen en el nivel de soporte social, tales como el estrato socioeconómico, el nivel de escolaridad y vivir en el mismo domicilio de la persona que se cuida, aspectos que deben ser considerados en futuras estrategias de intervención para esta población. Otra fortaleza fue la buena consistencia interna del instrumento utilizado. 
Dentro de las limitaciones de este estudio se encuentra que el muestreo no fue aleatorio, indicando entonces que los resultados aquí presentados serán aplicables a la muestra en estudio y extrapolables a población con características similares. En relación al instrumento, consideramos que una debilidad fue no haber incluido la dimensión de ayuda tangible, la cual hace parte del constructo de soporte social y aunque no es una función del equipo de salud dar este tipo de apoyo, sí debe tenerse en cuenta al momento al diseñarse intervenciones para fortalecer el soporte social en los cuidadores de personas con enfermedades crónicas.

Los resultados de este estudio dejan ver que la percepción de Soporte Social por parte de los cuidadores constituye un aspecto clave, no sólo para el buen desarrollo del rol de cuidador familiar, sino para su propio bienestar. Consideramos que este trabajo constituye una línea de base para el desarrollo de trabajos futuros, que permitan evaluar los determinantes del soporte social e implementar estrategias de intervención que contribuyan a mejorar el soporte y apoyo que se les brinda a los cuidadores. Es así que los miembros del equipo de salud, deben identificar las necesidades de apoyo de los cuidadores e implementar planes de cuidado que contribuyan a mejor el soporte social en ellos.

De igual manera, es necesario que se involucre al personal de enfermería en la ejecución de estrategias educativas $y$ de soporte que fomenten el afrontamiento de la enfermedad por parte los cuidadores y así mismo que las instituciones de salud teniendo en cuenta el incremento de las enfermedades crónicas capaciten al personal de forma adecuada para ofrecer apoyo emocional y espiritual.

\section{Conclusión}

Un bajo soporte social fue percibido por los cuidadores, en especial para la dimensión de interacción social. Este hallazgo es relevante teniendo en cuenta que el soporte social constituye un aspecto clave en el desempeño del rol de cuidador en personas con enfermedad crónica y las implicaciones que tiene el bajo soporte social, tanto para la persona enferma como para su cuidador.

\section{Financiación}

Este proyecto fue financiado por la Universidad Industrial de Santander, según convocatoria de la Vicerrectoría de Investigación y Extensión.

\section{Agradecimientos}

Hospital Universitario de Santander y los Cuidadores que aceptaron su participación. Al Grupo de Cuidado al Paciente Crónico y su Familia, de la Universidad Nacional de Colombia por permitirnos vincularnos a este proyecto.

Conflicto de intereses: Ninguno declarado.

\section{Referencias}

1. Menéndez J, Guevara A, Arcia N, León Díaz EM, Marín C, Alfonso JC. Enfermedades crónicas y limitación funcional en adultos mayores: estudio comparativo en siete ciudades de América Latina y el Caribe. Revista Panamericana de Salud Pública. 2005;17(5/6):353-61.

2. Sabate E, Organización Mundial de la Salud. Adherencia a los tratamientos a largo plazo: pruebas para la acción. Ginebra: OMS; 2004.

3. Organización Mundial de la Salud. Informe Mundial de la OMS. Prevención de las enfermedades crónicas: una inversión vital. Ginebra: OMS; 2005. Disponible en: http://www.who.int/chp/chronic_disease_report/part 1/es/print.html.

4. Organización Mundial de la Salud. Las enfermedades no transmisibles, un problema de salud de primer orden para el siglo XXI: Estadísticas Sanitarias Mundiales 2012. Ginebra: OMS; 2012.

5. Melgarejo LM, Algarra AJC, Rubio FM. La cronicidad y sus matices: estudio documental. Investigación en Enfermería: Imagen y Desarrollo. 2013;15(2):95-114.

6. Sánchez-Herrera B, Pinto-Afanador N. El reto de los cuidadores: familiares de personas en situación crónica de enfermedad. En: Universidad Nacional de Colombia, editores. Cuidado y práctica de enfermería. Bogotá, Colombia: Universidad Nacional de Colombia; 2000. p. 172-83.

7. Díaz-Álvarez JC, Pinilla-Alarcón M, Eslava-Albarracín DG, Padilla-Velasco MP. Soporte social con herramienta edumática para cuidadores familiares de personas con enfermedad crónica. Aquichan. 2014;14(3):364-87.

8. Correa Uribe BL, Romero Guevara SL, Camargo Ramírez MI. Características y percepciones de las cuidadoras de 
familiares con enfermedad crónica. REVISTA UIS HUMANIDADES. 2012;39(1):135-51.

9. Powers SE. The family caregiver program: Design and effectiveness of an education intervention. Home Healthcare Now. 2006;24(8):513-6.

10. Curcio Borrero CL. Soporte social informal, salud y funcionalidad en el anciano. Hacia la Promoción de la Salud. 2008;13(1):42-58.

11. Hilbert G. Measuring social support in chronic illness. In: Strickland O, Waltz C, editors. Measurement of Nursing Outcomes, Self Care and Coping Skills. Volume 3. New York: Springer Publishing Company; 1990. p. 79-96.

12. Vargas-Escobar LM. Marco para el Cuidado de la Salud en Situaciones de Enfermedad Crónica. Investigación en Enfermería: Imagen y Desarrollo. 2011;12(1):79-94.

13. Barrera-Ortiz L, Carrillo G, Chaparro-Díaz L, SánchezHerrera B. Cuidado de enfermería en situaciones de enfermedad crónica. Primera edición ed. Barrera-Ortiz L, Carrillo G, Chaparro-Díaz L, Sánchez-Herrera B, editors. Bogotá: Universidad Nacional de Colombia (Bogotá). Facultad de Enfermería; 2014. 254 p.

14. Atlanta. Cd. Epi Info Epidemiología en ordenadores v. $6.04 \mathrm{~d}$ ed. Atlanta, Georgia: Center for Disease Control and Prevention and World Health Organization; 2001.

15. Stata Corp LP. Stata: Release 12. In: LP SC, editor. 12 ed. College Station, TX: Stata Corp LP; 2011.

16. República de Colombia, Ministerio de Salud. Resolución no 008430 de 1993: Por la cual se establecen las normas científicas, técnicas y administrativas para la investigación en salud. Bogota: MinSalud; 1993.

17. Consejo de Organizaciones Internacionales de las Ciencias Médicas. Pautas éticas internacionales para la investigación biomédica en seres humanos. Ginebra: CIOMS; 2002.

18. Crespo-López M, López-Martínez J. El apoyo a los cuidadores de mayores dependientes: presentación del programa" cómo mantener su bienestar". Madrid, España: Ministerio de Trabajo y Asuntos Sociales. Secretaría de Estado de Servicios Sociales, Familias y Discapacidad. Instituto de Mayores y Servicios Sociales (IMSERSO); 2007. Disponible en: http://www.imserso.es/InterPresent2/groups/imsers o/documents/binario/apoyocuidadores.pdf.

19. Moreno ME, Náder A, de Mesa CL. Relación entre las características de los cuidadores familiares de pacientes con discapacidad y la percepción de su estado de salud. Avances en enfermería. 2004;22(1):27-38.

20. Vega Angarita O, González Escobar D. Apoyo social: elemento clave en el afrontamiento de la enfermedad crónica. Enfermeria global. 2009;8(2):1-11.

21. Williams P, Barclay L, Schmied V. Defining social support in context: a necessary step in improving research, intervention, and practice. Qualitative health research. 2004;14(7):942-60.

22. Sánchez-Herrera B, Carrillo-González GM, Barrera-Ortiz L, Chaparro-Díaz L. Carga del cuidado de la enfermedad crónica no transmisible. Aquichán. 2013;13(2):247-60.
23. Caro Dougnac D. Impacto económico de las enfermedades crónicas. Santiago de Chile: Universidad de Chile; 2014.

24. Carrillo GM, Chaparro Díaz L, Sánchez Herrera B. Carga del cuidado en cuidadores familiares de personas con enfermedad crónica en la región pacífica colombiana. Ciencia y enfermería. 2014;20(2):83-91.

25. Alwan A. Global status report on noncommunicable diseases 2010. Geneve: World Health Organization; 2010.

26. Quero Rufián A. Los cuidadores familiares en el Hospital Ruiz de Alda de Granada. Granada, España: Universidad de Granada; 2007.

27. Herrera A, Montalvo A, Flórez I, Romero E. Soporte social a cuidadores familiares de personas con enfermedad crónica en Cartagena. Aquichan. 2012;12(3):286-97.

28. Barrera-Ortiz L, Carrillo-González GM, Chaparro-Díaz L, Pinto-Afanador N, Sánchez-Herrera B. Soporte social con el uso de TIC's para cuidadores familiares de personas con enfermedad crónica. Revista de Salud Pública. 2011;13(3):446-57. 